\title{
Fatores de risco para recidiva de peso no pós-operatório tardio de cirurgia bariátrica
}

\author{
Risk factors for weight regain in the late postoperative of bariatric surgery
}

Adriane Granato Bardal ${ }^{\circledR} \bowtie$, Vanessa Ceccatto', Thais Regina Mezzomo²

${ }^{1}$ Centro Universitário Campos de Andrade (Uniandrade). Curitiba, PR.

${ }^{2}$ Universidade Positivo. Curitiba, PR.

\section{RESUMO}

Objetivos: Avaliar os fatores de risco para a recidiva de peso em indivíduos submetidos à cirurgia bariátrica.

Métodos: Estudo transversal utilizando-se questionário autoaplicável online. O questionário abordou fatores que poderiam estar associados à recidiva de peso após cirurgia bariátrica. Foram registrados peso, altura, sexo, idade, escolaridade, renda e assiduidade às consultas nutricionais no pré e no pós-operatório. Foram avaliados índice de massa corporal, perda de peso e perda do excesso de peso no pós-operatório imediato e após seis, 12 e 24 meses de pós-operatório, assim como no momento em que o participante respondeu ao questionário. O reganho de peso foi avaliado a partir do menor peso obtido após a cirurgia. O questionário Three Factor Eating Questionnaire-21 foi utilizado para classificar o comportamento alimentar em Alimentação Emocional, Restrição Cognitiva ou Descontrole Alimentar.

Resultados: Foram respondidos 46 questionários por indivíduos que realizaram cirurgia bariátrica, com média de idade de $38 \pm 8,78$ anos e mediana de três anos de pós-operatório. Quarenta e três $(93,5 \%)$ participantes eram do sexo feminino. As médias de perda do excesso de peso e a perda de peso atual foram de $78,56 \pm 16,12 \%$ e $35,24 \pm 8,19 \%$, respectivamente. A média do índice de massa corporal atual era de $27,35 \pm 3,98 \mathrm{~kg} / \mathrm{m}^{2}$. Alimentação Emocional foi encontrada em 18 (39,13\%), Restrição Cognitiva em 24 (52,17\%) e Alimentação Emocional associada à Restrição Cognitiva em 4 (8,69\%) dos entrevistados. A recidiva de peso ocorreu em 18 (39\%) dos indivíduos, a partir principalmente dos 12 meses após a cirurgia. Observou-se que a renda familiar mensal superior a 4000 reais e a frequência a consultas com nutricionista inferior a três vezes no pré-operatório estiveram associadas a maior chance de recidiva de peso.

Conclusões: Nessa amostra, a baixa assiduidade às consultas nutricionais no pré-operatório, bem como a renda familiar elevada foram fatores de risco para recidiva de peso no pós-operatório tardio de cirurgia bariátrica.

DESCRITORES: cirurgia bariátrica; obesidade; programas de nutrição.

\section{ABSTRACT}

Aims: To evaluate risk factors for weight regain in patients undergoing bariatric surgery.

Methods: Cross-sectional study using self-administered online questionnaire. The questionnaire asked about factors that can be associated with weight regain after bariatric surgery. Weight, height, sex, age, education, income, and nutrition appointment attendance before and after surgery were registered. Body mass index, weight loss, and loss of excess weight in the immediate postoperative period and at 6,12 , and 24 months postoperatively, as well as at the time of completing the questionnaire, were evaluated. Weight regain was evaluated according to the lowest weight achieved since surgery. The Three Factor Eating Questionnaire-21 was used to classify eating behavior into Emotional Eating, Cognitive Dietary Restraint, or Uncontrolled Eating.

Results: The questionnaire was answered by 46 individuals who underwent bariatric surgery, with a mean age of $38 \pm 8.78$ years and a median of three postoperative years. Forty-three (93.5\%) patients were female. The mean loss of excess weight and current weight loss were $78.56 \pm 16.12 \%$ and $35.24 \pm 8.19 \%$, respectively. The current body mass index was $27.35 \pm 3.98 \mathrm{~kg} / \mathrm{m}^{2}$. Emotional eating was observed in $18(39.13 \%)$, cognitive restraint in $24(52.17 \%)$, and emotional eating associated with cognitive restraint in $4(8.69 \%)$ of the respondents. Eighteen individuals (39\%) regained weight, especially after 12 months. A monthly family income greater than 4000 Brazilian Reals and nutrition appointments fewer than three in the preoperative period were associated with a larger probability of weight regain.

Conclusions: In this sample, low nutrition appointment attendance in the preoperative period and a high family income were risk factors for weight regain in the late postoperative period of bariatric surgery.

KEY WORDS: bariatric surgery; obesity; nutrition programs. 
Abreviaturas: IMC, índice de massa corporal; PP, perda de peso; $\%$ PP, percentual de perda de peso; PEP, perda do excesso de peso; $\%$ PEP, percentual de perda do excesso de peso; TFEQ-21, Three Factor Eating Questionnaire.

\section{INTRODUÇÃO}

A obesidade, doença crônica caracterizada pelo acúmulo excessivo de energia sob a forma de triglicérides no tecido adiposo, tem como principais causas o consumo alimentar excessivo, o sedentarismo, tendências genéticas e distúrbios hormonais $[1,2]$. As importantes transformações demográficas, econômicas e tecnológicas, além do aumento da expectativa de vida e redução de mortes por doenças infecto-parasitárias, também contribuem para essa condição que atinge mais de 600 milhões de adultos e 42 milhões de crianças em todo o mundo $[3,4]$.

A cirurgia bariátrica vem se mostrando como o tratamento mais efetivo para a perda de peso e controle das comorbidades da obesidade, quando ocorre insucesso nos tratamentos conservadores $[2,5,6]$. No Brasil, tem-se observado considerável aumento no número de cirurgias bariátricas realizadas [2]. Contudo, apesar da eficácia da cirurgia bariátrica na perda de peso, a recuperação deste pode ocorrer após uma perda satisfatória ou mesmo antes de obtê-la, conforme já demonstrado em diversos estudos [7-10].

Estima-se que entre 5 a $60 \%$ dos indivíduos submetidos à cirurgia bariátrica são reoperados, por complicações ou devido a resultados insatisfatórios [10]. Aproximadamente 50\% dos pacientes irão retornar ao seu peso inicial após cinco anos do procedimento cirúrgico [11]. Esses resultados demonstram que o principal desafio do tratamento da obesidade não é a perda de peso, mas a sua manutenção em longo prazo, o que aumenta os riscos à saúde dos indivíduos, principalmente no âmbito emocional, e os custos em saúde [12-14].

Por outro lado, muitos indivíduos submetidos à cirurgia bariátrica apresentam êxito na manutenção do peso. Fatores comportamentais, como altos níveis de atividade física, baixo consumo calórico e lipídico, baixo índice glicêmico dos alimentos consumidos e automonitorização frequente do peso; fatores emocionais como redução da desinibição e satisfação com os resultados alcançados; alguns traços de personalidade, como a baixa procura por novidades e a interação paciente-terapeuta; intervenções farmacológicas; e fatores hormonais e moleculares, têm sido associados com a manutenção do peso em longo prazo $[11,14,15]$.
Conhecer os fatores envolvidos na recidiva do ganho de peso pode auxiliar na manutenção do excesso de peso perdido e precisam ser analisados criteriosamente. Dessa forma, foi objetivo desse estudo avaliar os fatores de risco para a recidiva de peso em indivíduos submetidos à cirurgia bariátrica.

\section{MÉTODOS}

Foi realizado um estudo transversal com indivíduos submetidos à cirurgia bariátrica, utilizandose um questionário autoaplicável online. $\mathrm{O}$ projeto foi aprovado pelo Comitê de Ética em Pesquisa do Centro Universitário Campos de Andrade sob parecer consubstanciado de número 1.544.419.

Os critérios de inclusão no estudo foram indivíduos submetidos a qualquer modalidade de cirurgia bariátrica com no mínimo 24 meses de póscirurgia, com idade superior a 18 anos. O convite para a participação no estudo foi realizado por meio eletrônico, via rede social. Os questionários online foram disponibilizados em grupos de redes sociais de indivíduos que realizaram cirurgia bariátrica, residentes em Curitiba, Paraná. O termo de consentimento livre e esclarecido foi disponibilizado na primeira página do questionário online, e somente após a concordância deste o participante tinha acesso ao questionário na íntegra. Caso o participante não concordasse com o termo, o questionário era encerrado automaticamente.

O questionário incluiu dados como sexo, data de nascimento, escolaridade, renda familiar, tipo de cirurgia realizada, tempo de pós-operatório, altura e peso no pré-operatório; o primeiro peso no pósoperatório, bem como o peso aos 6, 12, 18 e 24 meses, além do peso atual no momento da pesquisa.

Foi calculado o índice de massa corporal (IMC) empregando a fórmula que utiliza a razão peso (em quilos) sobre altura (em metros) ao quadrado, e o resultado foi classificado de acordo com o Ministério da Saúde do Brasil [16]. O peso ideal foi calculado utilizando-se o IMC limítrofe de eutrofia de $24,9 \mathrm{~kg} / \mathrm{m}^{2}$ $\mathrm{e}$, então, utilizou-se a seguinte fórmula para o cálculo do percentual de perda do excesso de peso (\%PEP): (Peso usual - Peso atual) x 100/(Peso usual - P ideal) [17]. $\mathrm{O}$ percentual de perda de peso $(\% \mathrm{PP})$ foi calculado por meio da fórmula: (Peso usual - Peso atual) x 100/Peso usual. O reganho de peso foi calculado como um ganho de peso superior a $10 \%$ sobre o menor valor de peso corporal relatado pelo indivíduo no pós-operatório [2].

A assiduidade às consultas nutricionais foi avaliada de acordo com o número de consultas com uma equipe de nutrição a que o entrevistado informava 
ter comparecido no pré-operatório, e o número de participações em consultas e/ou grupos de nutrição no primeiro e no segundo ano de pós-operatório.

Para a avaliação do comportamento alimentar foi utilizado o questionário Three Factor Eating Questionnaire (TFEQ-21) com 21 questões [18]. O TFEQ-21 classifica os comportamentos em a) Alimentação Emocional, que caracteriza a tendência do indivíduo a aumentar a ingestão alimentar em situações desafiadoras como ansiedade, estresse, solidão e nervosismo; b) Restrição Cognitiva, que caracteriza um conjunto de regras e proibições alimentares com intuito de manter o peso saudável; e c) Descontrole Alimentar, caracterizado pela perda de controle sobre a porção ingerida ou sobre a quantidade calórica da porção [19]. As questões de 1 a 16 apresentavam quatro alternativas para marcação pelo participante, com pontuação de 1 a 4 , sendo 4 pontos para a primeira alternativa e 1 ponto para a última alternativa. Para as questões de 17 a 20, a pontuação deu-se ao contrário, sendo 1 ponto para a primeira alternativa e 4 pontos para a última alternativa e, para a questão 21 , a qual apresentava uma escala de 8 pontos, quantificou-se conforme: alternativas 1 e 2 com pontuação 1 , alternativas 3 e 4 com pontuação 2, alternativas 5 e 6 com pontuação 3 e alternativas 7 e 8 com pontuação 4 [20]. A quantificação foi calculada pelas pesquisadoras, após o participante ter selecionado as alternativas no questionário. Para detecção de alimentação emocional, se três ou mais itens dentre as questões 2, 4, 7, 10, 14 e 16 foram assinalados pelo participante, realizou-se a média da pontuação desses itens marcados e multiplicou-se por seis. Para determinação de comportamento restritivo, se três ou mais itens dentre as questões $1,5,11,17,18$ e 21 foram assinalados, realizou-se a média da pontuação e multiplicou-se por seis. E, para avaliar o descontrole alimentar, se cinco ou mais itens, dentre as questões $6,8,9,12,13,15,19$ e 20 foram assinalados, realizou-se a média da pontuação desses itens e multiplicou-se por nove. O resultado obtido foi transformado para a escala de 0 a 100 pontos conforme a fórmula: [(resultado das questões multiplicadas pelo fator - menor escore possível) / escala de pontos possíveis*100], com o auxílio do Quadro 1 [19]. Altos escores indicam presença de alimentação emocional, de comportamento restritivo e descontrole alimentar [18].

Os resultados foram descritos por meio de distribuição de frequência simples, média e desvio padrão, e compilados no programa Microsoft Excel®. Para as análises estatísticas foram utilizados o teste $\mathrm{t}$ de Student para variáveis quantitativas e o quiquadrado para as variáveis qualitativas, com o auxílio do programa Paleontological Statistics versão 2.16. O nível de significância adotado foi de $p<0,05$.

\section{RESULTADOS}

Foram analisados 46 questionários de indivíduos que realizaram cirurgia bariátrica, com média de idade de 38 $\pm 8,78$ anos, sendo 43 (93,5\%) do sexo feminino. O momento de resposta ao questionário teve uma mediana de três anos após a cirurgia. O tipo de cirurgia bariátrica realizada pelos avaliados foi do tipo cirurgia mista em $88,9 \%$ dos casos, seguida de técnica restritiva $(8,9 \%)$ e disabsortiva $(2,2 \%)$.

Entre os avaliados, $32(71,1 \%)$ tinham ensino superior completo e $24(52,2 \%)$ apresentavam renda familiar mensal inferior a R\$4.000,00. Nenhum dos entrevistados relatou renda familiar inferior a $\mathrm{R} \$$ $1.000,00$.

Dentre os $18(39,13 \%)$ pacientes que apresentaram recidiva de peso, o reganho médio de peso foi de 9,6kg. A Figura 1 mostra a evolução do peso corporal, do \%PP, do IMC e do \%PEP dos indivíduos avaliados após a realização da cirurgia bariátrica. Observa-se o reganho de peso após os 12 meses de pós-cirurgia, quando a \% $\%$ e a \%PEP passam a diminuir.

Quanto aos fatores de risco, observou-se que renda familiar mensal superior a $\mathrm{R} \$ 4.000,00$ e três consultas nutricionais ou menos foram associados significativamente com a recidiva de peso (Tabela $\mathbf{1}$ ).

Quadro 1. Fórmulas de transformação de escores para a escala de 0 a 100 pontos, referente à avaliação do comportamento alimentar pelo questionário Three Factor Eating Questionnaire (TFEQ-21).

\begin{tabular}{|l|c|c|c|}
\hline \multicolumn{1}{|c}{} & \multicolumn{1}{c}{$\begin{array}{c}\text { Menor/maior } \\
\text { pontuação possível }\end{array}$} & $\begin{array}{c}\text { Escala de pontos } \\
\text { possíveis }\end{array}$ & Fórmula para escore \\
\cline { 2 - 4 } Descontrole Alimentar & $9 / 36$ & 27 & [(resultado das questões multiplicadas pelo fator -9$) / 27] * 100$ \\
\hline Comportamento Restritivo & $6 / 24$ & 18 & $[$ [(resultado das questões multiplicadas pelo fator -6$) / 18] * 100$ \\
\hline Alimentação Emocional & $6 / 24$ & 18 & $[$ [resultado das questões multiplicadas pelo fator -6$) / 18] * 100$ \\
\hline
\end{tabular}

Fonte: adaptado de Tholin et al. [18] e Natacci [19]. 


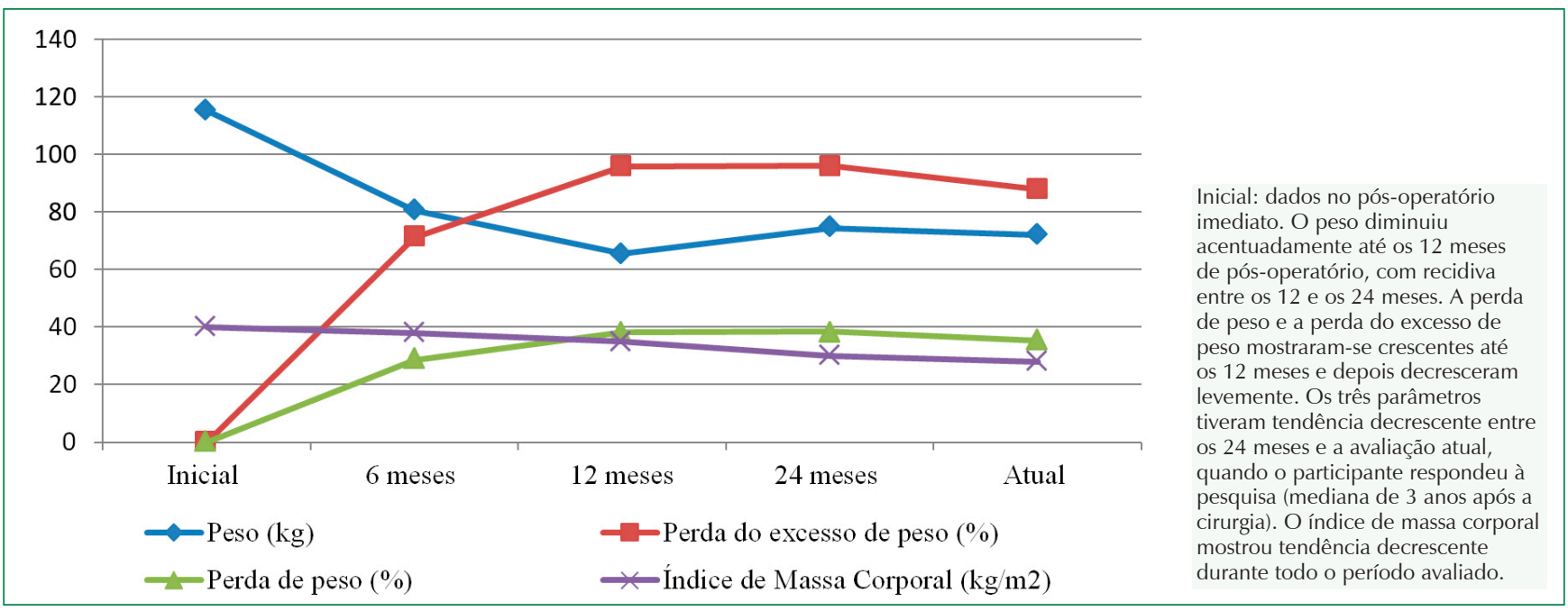

Figura 1. Evolução do peso corporal, da perda de peso, da perda de excesso de peso e do índice de massa corporal em 46 indivíduos no pós-operatório de cirurgia bariátrica. Curitiba, Paraná, 2015.

Tabela 1. Fatores de risco para a recidiva de peso corporal no pós-operatório tardio de indivíduos submetidos à cirurgia bariátrica. Curitiba, Paraná, 2015.

\begin{tabular}{|c|c|c|c|}
\hline & $\begin{array}{c}\text { Sem recidiva de peso } \\
\mathrm{n}(\%)\end{array}$ & $\begin{array}{c}\text { Com recidiva de peso } \\
\mathbf{n}(\%)\end{array}$ & $\mathbf{p}$ \\
\hline \multicolumn{4}{|l|}{ Sexo } \\
\hline Feminino & $25(58,1)$ & $18(41,9)$ & \multirow[t]{2}{*}{$0,14^{*}$} \\
\hline Masculino & $3(100)$ & $0(0)$ & \\
\hline \multicolumn{4}{|l|}{ Idade } \\
\hline Entre 18 e 30 anos & $5(62,5)$ & $3(37,5)$ & \multirow[t]{2}{*}{$0,91^{*}$} \\
\hline Superior a 30 anos & $23(60,52)$ & $15(39,43)$ & \\
\hline \multicolumn{4}{|l|}{ Escolaridade } \\
\hline Superior completo & $12(50)$ & $12(50)$ & \multirow{2}{*}{$0,08^{*}$} \\
\hline Outros & $15(75)$ & $5(25)$ & \\
\hline \multicolumn{4}{|l|}{ Renda familiar mensal } \\
\hline Entre $R \$ 1.001,00$ e $R \$ 4.000,00$ & $18(75)$ & $6(25)$ & \multirow{2}{*}{$0,04 *$} \\
\hline Mais de $\mathrm{R} \$ 4.000,00$ & $10(45,5)$ & $12(54,5)$ & \\
\hline \multicolumn{4}{|c|}{ Frequência de consultas com nutricionista no pré-operatório } \\
\hline Até 3 & $6(30,0)$ & $14(70,0)$ & \multirow{2}{*}{$0,008^{*}$} \\
\hline Mais de 3 & $18(69,2)$ & $8(30,8)$ & \\
\hline \multicolumn{4}{|c|}{ Frequência de consultas no $1^{\circ}$ ano de pós-operatório } \\
\hline Até 3 & $10(50)$ & $10(50)$ & \multirow{2}{*}{$0,10^{*}$} \\
\hline Mais de 3 & $19(73,07)$ & $7(26,92)$ & \\
\hline \multicolumn{4}{|c|}{ Frequência de consultas no $2 \stackrel{0}{ }$ ano de pós-operatório } \\
\hline Até 3 & $22(62,85)$ & $13(37,15)$ & \multirow{2}{*}{$0,62^{*}$} \\
\hline Mais de 3 & $6(54,54)$ & $5(45,46)$ & \\
\hline \multicolumn{4}{|l|}{ Frequência de consultas (geral) no pós-operatório } \\
\hline Até 3 & $10(50)$ & $10(50)$ & \multirow[t]{2}{*}{$0,05^{*}$} \\
\hline Mais de 3 & $20(76,92)$ & $6(23,08)$ & \\
\hline \multicolumn{4}{|l|}{ Comportamento alimentar avaliado pelo TFEQ-21 } \\
\hline Alimentação Emocional & $7(38,88)$ & $11(61,12)$ & \multirow{4}{*}{$\begin{array}{l}0,47^{+} \\
0,56^{+}\end{array}$} \\
\hline Restrição Cognitiva & $19(79,16)$ & $5(20,84)$ & \\
\hline Alimentação Emocional+Restrição Cognitiva & $2(50)$ & $2(50)$ & \\
\hline Descontrole Alimentar & $0(0)$ & $0(0)$ & \\
\hline
\end{tabular}

TFEQ-21, Three Factor Eating Questionnaire.

* Qui-quadrado; ${ }^{+}$Teste t de Student. 
Em relação ao comportamento alimentar avaliado pelo TFEQ-21, todos os indivíduos avaliados apresentaram algum tipo de alteração do comportamento alimentar, com predominância do tipo Restrição Cognitiva. Neste estudo não se observou associação de alteração do comportamento alimentar com presença ou ausência de recidiva de peso (Tabela 1).

\section{DISCUSSÃO}

A cirurgia bariátrica é o método mais efetivo para o tratamento da obesidade mórbida, porém apresenta risco de complicações em longo prazo $[2,10]$. Para minimizar e tratar precocemente as complicações oriundas da cirurgia recomenda-se o acompanhamento clínico e nutricional por equipe multidisciplinar [21].

Corroborando com os resultados desse trabalho, a predominância de mulheres nos casos de cirurgia bariátrica no Brasil tem sido verificada consistentemente, embora com variação nas proporções [22-24]. Mulheres de todos os grupos étnicos apresentam maior probabilidade de se submeterem a cirurgia bariátrica do que homens, talvez pela maior motivação de perda de peso por razões estéticas e sociais $[23,25]$.

A restrição no consumo alimentar provocada pela cirurgia bariátrica desencadeia grande perda de peso, considerada como um dos principais resultados para um bom desfecho, levando à melhora das condições clínicas do indivíduo [17]. A PEP é expressiva no primeiro mês pós-cirúrgico e há tendência de se acentuar até o sexto mês, quando começa a estabilizar, fato esse concretizado entre o $12^{\circ}$ e $18^{\circ}$ mês de pós-operatório [26]. É consenso na literatura que o tratamento cirúrgico da obesidade obtém sucesso quando o \%PEP é de no mínimo 50\%, com manutenção por um longo período [17]. Neste estudo, verificou-se um \%PEP médio bem acima desse valor. É importante destacar que esses valores não consideraram o tipo de cirurgia bariátrica realizada.

Os fatores que contribuem para a perda de peso após a cirurgia bariátrica compreendem fatores mecânicos, como redução da capacidade gástrica, bem como fatores hormonais. Estes últimos incluem diminuição da grelina, conhecida como "hormônio da fome", diminuição da resistência à leptina, conhecida como "hormônio da regulação da saciedade", assim como possível aumento de incretinas, como o peptídeo YY e o glucagon-like peptídeo-1, possivelmente em função do conteúdo gástrico ser movido mais rapidamente para o intestino, estimulando as células $\mathrm{L}$ jejunais a liberarem essas incretinas, melhorando o controle da fome e da saciedade [27-29].

Apesar da eficácia da cirurgia bariátrica, a recuperação do peso pode ocorrer em longo prazo [6-10]. Neste estudo, a baixa assiduidade às consultas nutricionais no pré-operatório refletiu-se significativamente na recidiva de peso corporal. Este resultado sugere que o indivíduo com maior frequência às consultas nutricionais antes de ser submetido à cirurgia pode obter melhor educação alimentar e nutricional, contribuindo para resultados mais duradouros de perda e manutenção de peso corporal. Além disso, a pessoa que adere a um programa prévio à cirurgia tende a ter maior motivação para um estilo de vida saudável, o qual é primordial para a manutenção do peso, envolvendo a multidisciplinaridade de alimentação saudável, atividade física e acompanhamento psicológico $[10,17,30]$.

Por outro lado, no presente estudo não se verificou associação da frequência de consultas nutricionais no pós-operatório com a recidiva de peso, provavelmente devido ao baixo número de indivíduos que mantiveram acompanhamento nutricional após a cirurgia. $\mathrm{O}$ acompanhamento nutricional no pós-operatório da cirurgia bariátrica visa favorecer a identificação e a intervenção precoce na perda de peso insuficiente ou na recidiva de peso, assim como em deficiências nutricionais, contribuindo favoravelmente para o tratamento cirúrgico [21].

O nível socioeconômico é um dos principais determinantes de saúde, e o acesso a uma dieta saudável por indivíduos de alto poder aquisitivo é um dos mecanismos que explicam as diferenças sociais em saúde [31]. Diversos estudos demonstram o consumo de dietas altamente calóricas e menor acesso à alimentação adequada e saudável em níveis socioeconômicos menores [21,32]. Entretanto, neste estudo, resultado inesperado foi observado na recidiva de peso em indivíduos com renda familiar mensal superior a 4.000 reais. Sugere-se que esse resultado seja devido à maior possibilidade de acesso a alimentos altamente palatáveis e densamente energéticos, os quais têm alta estimulação hedônica e induzem à hiperalimentação, mesmo na ausência de fome [33]. O retorno a maus hábitos alimentares após longo prazo da realização da cirurgia bariátrica pode facilitar o aumento do tamanho da anastomose gastrojejunal e assim, promover a volta a um consumo energético excessivo [8]. Além disso, padrões comportamentais proeminentes no mundo ocidental, tais como a privação do sono, o hábito de realizar refeições em frente à televisão, o uso constante dos dispositivos eletrônicos, bem como o consumo abusivo de álcool, 
são fatores associados com a recompensa alimentar e a adiposidade [34].

Apesar de o nível de escolaridade não ter exercido influência na recidiva de peso neste estudo, observase que mais da metade da população avaliada apresentava ensino superior completo, o que pressupõe entendimento do contexto de vida saudável e maior facilidade de adesão às recomendações recebidas [21].

Neste estudo, o comportamento alimentar avaliado pelo questionário TFEQ-21 mostrou-se alterado em todos os indivíduos avaliados, porém, não se observou associação significativa entre recidiva de peso e situações desafiadoras, como ansiedade, estresse e solidão, proibições alimentares ou quantidade de alimentos consumidos.

O presente estudo apresenta limitações, como a característica de autopreenchimento de dados, gerando, por conseguinte, viés de memória, omissão de informações, dados sub ou superestimados e a exacerbação de sentimentos referentes ao comportamento em relação ao alimento. Ainda, este estudo não avaliou a prática de atividade física e o consumo alimentar dos indivíduos.

Apesar das limitações, pode-se concluir que baixa assiduidade às consultas nutricionais no pré-operatório e renda familiar elevada representaram fatores de risco para recidiva de peso no pós-operatório tardio de cirurgia bariátrica, na amostra estudada. Para a manutenção do peso após a cirurgia bariátrica pode ser necessária uma abordagem multidisciplinar, com associação de diversos tratamentos, envolvendo profissionais das áreas de medicina, nutrição, educação física e psicologia. Estudos sobre recidiva de peso identificam diversos fatores envolvidos e apontam para a necessidade de mais colaborações científicas que elucidem esses fatores, de forma a garantir os esperados resultados duradouros da cirurgia bariátrica.

\section{NOTA}

Declaração de conflitos de interesse

Os autores declaram não haver conflitos de interesse relevantes ao conteúdo deste estudo.

\section{REFERÊNCIAS}

1. Fagundes ALN, Ribeiro DC, Naspitz L, Garbelini LEB, Vieira JKP, Silva AP, Lima VO, Fagundes DJ, Compri PC, Yára J. Prevalência de sobrepeso e obesidade em escolares da região de Parelheiros do município de São Paulo. Rev Paul Pediatr. 2008;26(3):212-7. http://dx.doi.org/10.1590/S0103-05822008000300003

2. Sociedade Brasileira de Cirurgia Bariátrica e Metabólica. Número de Cirurgias Bariátricas realizadas no Brasil [Internet]. São Paulo: SBCBM; c2011 [cited 2015 Nov 30]. Avaliable from: http://www.sbcb.org.br/imprensa.php?menu=3

3. Burgos G. Nutrição em cirurgia bariátrica. Rio de Janeiro: Rubio; 2014.

4. World Health Organization. Obesity and Overweight [Internet]. Genebra: WHO; 2015 [cited 2015 Aug 08]. Available from: http://www. who.int/mediacentre/factsheets/fs311/en/

5. Buchwald H, Williams SE. Bariatric surgery worldwide 2003. Obes Surg. 2004;14(9):1157-64. http://dx.doi.org/10.1381/0960892042387057

6. Berti LV, Campos J, Ramos A, Rossi M, Szego T, Cohen R. Posição da SBCBM - Nomeclatura e definições para os resultados em cirurgia bariátrica e metabólica. Arq Bras Cir Dig. 2015;28(Supl.1):2. http://dx.doi.org/10.1590/S0102-6720201500S100002

7. Al-Bader I, Khoursheed M, Al Sharaf K, Mouzannar DA, Ashraf A, Fingerhut A. Revisional Laparoscopic Gastric Pouch Resizing for Inadequate Weight Loss After Roux-en-Y Gastric Bypass. Obes Surg. 2015;25(7):1103-8. http://dx.doi.org/10.1007/s11695-015-1579-9

8. Cambi MPC, Marchesini SD, Baretta GAP. Post-bariatric surgery weight regain: evaluation of nutritional profile of candidate patients for endoscopic argon plasma coagulation. Arq Bras Cir Dig. 2015;28(1):40-3. http://dx.doi.org/10.1590/S0102-67202015000100011

9. Cesana G, Uccelli M, Ciccaresse F, Carrieri D, Castello G, Olmi S. Laparoscopic re-sleeve gastrectomy as a treatment of weight regain after sleeve gastrectomy. World J Gastrointest Surg. 2014;27(6):101-6.

10. Daigle CR, Aminian A, Romero-Talamás H, Corcelles R, Mackey J, Rogula T, Brethauer SA, Schauer PR. Outcomes of a Third Bariatric Procedure for Inadequate Weight Loss. JSLS. 2014 July-Sept;18(3). pii: e2014.00117. http://dx.doi.org/10.4293/JSLS.2014.00117

11. Blomain ES, Dirhan DA, Valentino MA, Kim GW, Waldman SA. Mechanisms of Weight Regain following Weight Loss. ISRN Obes. 2013;2013:210524. http://dx.doi.org/10.1155/2013/210524

12. Annesi JJ. Psychosocial predictors of decay in healthy eating and physical activity improvements in obese women regaining lost weight: translation of behavioral theory into treatment suggestions. Transl Behav Med. 2016;6(2):169-78. http://dx.doi.org/10.1007/s13142-0160401-0

13. Hollywood A, Ogden J, Hashemi M. A randomised control trial assessing the impact of an investment based intervention on weight-loss, beliefs and behaviour after bariatric surgery: study protocol. BMC Obes. 2015 Mar 21;2:18. http://dx.doi.org/10.1186/s40608-015-0048-2

14. Montesi L, El Ghoch M, Brodosi L, Calugi S, Marchesini G, Dalle Grave R. Long-term weight loss maintenance for obesity: a multidisciplinary approach. Diabetes Metab Syndr Obes. 2016 Feb 26;9:37-46. https://dx.doi.org/10.2147/DMSO.S89836 
15. Anastasiou CA, Karfopoulou E, Yannakoulia M. Weight regaining: from estatistics and behaviors to physiology and metabolism. Metabolism. 2015;64(11):1395-407. http://dx.doi.org/10.1016/j.metabol.2015.08.006

16. Ministério da Saúde (BR). Orientações para a coleta e análise de dados antropométricos em serviços de saúde. Norma Técnica do Sistema de Vigilância Alimentar e Nutricional - SISVAN. Brasília: Ministério da Saúde; 2011.

17. Novais PFS, Rasera Junior, I, Leite CVS, Oliveira MRM. Evolução e classificação do peso corporal em relação aos resultados da cirurgia bariátrica: derivação gástrica em Y de Roux. Arq Bras Endocrinol Metab. 2010; 54(3):303-10. http://dx.doi.org/10.1590/S000427302010000300009

18. Tholin S, Rasmussen F, Tynelius P, Karlsson J. Genetic and environmental influences on eating behavior: the swdish young male twins study. Am J Clin Nutr. 2005;81(3):564-9.

19. Natacci LC. The three fator eating questionnarie-R21 (TFEQ-R21): tradução, aplicabilidade, comparação a um questionário semiquantitativo de frequência de consumo alimentar e a parâmetros antropométricos [dissertation]. [São Paulo]: Faculdade de Medicina da Universidade de São Paulo; 2009. Available from: http://www.teses.usp.br/teses/disponiveis/5/5160/tde-06042010-174236/pt-br.php

20. Cappelleri JC, Bushmakin AG, Gerber RA, Leidy NK, Sexton CC, Lowe MR, Karlsson J. Psychometric analysis of the Three-Factor Eating Questionnaire-R21: results from a large diverse sample of obese and non-obese participants. Int J Obes (Lond). 2009 June;33(6):611-20. http://dx.doi.org/10.1038/ijo.2009.74

21. Scabim VM, Eluf-Neto J, Tess BH. Adesão ao seguimento nutricional ambulatorial pós-cirurgia bariátrica e fatores associados. Rev Nutr. 2012;25(4):497-506. http://dx.doi.org/10.1590/S1415-52732012000400007

22. Garrido Júnior AB. Cirurgia em obesos mórbidos - experiência Pessoal. Arq Bras Endocrinol Metab. 2000;44(1):106-10. http://dx.doi. org/10.1590/S0004-27302000000100017

23. Hintze LJ, Bevilaqua CA, Pimentel EB, Nardo Junior N. Cirurgia bariátrica no Brasil. Rev Ciênc Méd. 2011;20(3-4):87-99.

24. Pareja JC, Pilla VF, Callejas-Neto F, Coelho-Neto JS, Chaim EA, Magro, DO. Gastroplastia redutora com bypass gastrojejunal em Y-deRoux: conversão para bypass gastrointestinal distal por perda insuficiente de peso - experiência em 41 pacientes. Arq. Gastroenterol. 2005;42(4):196-200. http://dx.doi.org/10.1590/S0004-28032005000400002

25. Mazzeo SE, Saunders R, Mitchell KS. Binge eating among African American and Caucasian bariatric surgey candidates. Eat Behav. 2005;6(3):189-96. http://dx.doi.org/10.1016/j.eatbeh.2004.12.001

26. Faria OP, Pereira VA, Gangoni CMC, Lins RD, Leite S, Rassi V, Arruda SLM. Obesos mórbidos tratados com gastroplastia redutora com bypass gástrico em Y de Roux: análise de 160 pacientes. Brasília Med. 2002;39(1/4):26-34.

27. Valverde I, Puente J, Martin-duce A, Molina KL, Lozano O, Sancho V, Villanueva-Pe-acarillo ML. Changes in glucagonlike peptide 1 (GLP-1) secretion after biliopancreatic diversion or vertical banded gastroplasty in obese subjects. Obes Surg. 2005; 15:(3)87-97. http:// dx.doi.org/10.1381/0960892053576613

28. Major P, Matłok M, Pędziwiatr M, Migaczewski M, Zub-Pokrowiecka A, Radkowiak D, Winiarski M, Zychowicz A, Fedak D, Budzyński A. Changes in levels of selected incretins and appetite-controlling hormones following surgical treatment for morbid obesity. Wideochir Inne Tech Maloinwazyjne. 2015 Sep;10(3):458-65. http://dx.doi.org/10.5114/wiitm.2015.54003

29. Silva RF, Kelly EO. Reganho de peso após o segundo ano do Bypass gástrico em Y de Roux. Com Ciências Saúde. 2013;24(4):341-50.

30. Odom J, Zalesin KC, Washington TL, Miller WW, Hakmeh B, Zaremba DL, Altattan M, Balasubramaniam M, Gibbs DS, Krause KR, Chengelis DL, Franklin BA, McCullough PA. Behavioral Predictors of Weight Regain After Bariatric Surgery. Obes Surg. 2010 Mar;20(3):349-56. http://dx.doi.org/10.1007/s11695-009-9895-6

31. Mayen AL, Marques-Vidal P, Paccaud F, Bovet P, Stringhini S. Socioeconomic determinants of dietary patterns in low- and middle-income countries: a systematic review. Am J Clin Nutr. 2014;100(6):1520-31. http://dx.doi.org/10.3945/ajcn.114.089029

32. Damon N, Drewnowski A. Does social class predict diet quality? Am J Clin Nutr. 2008;87(5):1107-17.

33. Sawaya AL, Leandro CG, Waitzberg DL. Fisiologia da Nutrição na Saúde e na Doença - Da Biologia Molecular ao Tratamento. São Paulo: Atheneu; 2013

34. Chapman CD, Benedict C, Brooks SJ, Birgir Schiöth H. Lifestyle determinants of the drive to eat: a meta-analysis. Am J Clin Nutr. 2012;96(3):492-7. http://dx.doi.org/10.3945/ajen.112.039750 